\title{
Comments on: High-dimensional simultaneous inference with the bootstrap
}

\author{
Matthias Löffler ${ }^{1} \cdot$ Richard Nickl $^{1}$ (D)
}

Published online: 9 October 2017

(C) Sociedad de Estadística e Investigación Operativa 2017

We would like to congratulate Ruben Dezeure, Peter Bühlmann and Cun-Hui Zhang for a stimulating and methodologically important contribution to the field of highdimensional statistics. They propose a bootstrap methodology to infer the distribution of the statistic $\max _{j \in G}\left|\hat{\beta}_{j}-\beta_{j}\right| / \hat{\sigma}_{j}$ for any subset $G \subseteq\{1 \ldots, p\}$, which particularly allows the construction of simultaneous $\ell_{\infty}$-confidence 'bands' for the whole parameter $\beta$. Together with the suggested variance estimator this offers robustness against misspecification of the error distribution and takes possible heteroscedasticity into account. For the proofs multiplier bootstrap ideas from Chernozhukov et al. (2013) are employed with technical virtuosity.

Here we discuss the potential shortfalls of this method for inference for relevant functionals of $\beta$. Our main caveat is that one should not be misled by the fact that one has a confidence region for the whole parameter and conclude that statistical inference for every aspect of $\beta$ is feasible. Even for simple linear functionals the plug-in confidence set is possibly not useful. This is due to the fact that in the highdimensional setting not all norms are equivalent.

Let us illustrate this in the prediction problem in random design: one observes $\left(Y_{1}, X_{1}\right), \ldots,\left(Y_{n}, X_{n}\right)$ where for simplicity $X_{i}$ are $i . i . d$. Gaussian with unit variance, and is interested in predicting the linear functional $E\left[Y_{n+1} \mid X_{n+1}\right]=X_{n+1}^{T} \beta^{0}$ for some design vector $X_{n+1}$. The plug-in confidence set for $X_{n+1}^{T} \beta$ is

This comment refers to the invited paper available at: doi:10.1007/s11749-017-0554-2.

Richard Nickl

r.nickl@statslab.cam.ac.uk

Matthias Löffler

m.loffler@statslab.cam.ac.uk

1 Statistical Laboratory, Centre for Mathematical Sciences, University of Cambridge, Wilberforce Road, Cambridge CB3 0WB, United Kingdom 


$$
C_{n}:=\left\{X_{n+1}^{T} \beta: \beta \in C_{n}^{\beta}\right\}
$$

where the construction of the confidence set $C_{n}^{\beta}$ is given by Theorem 3 in Dezeure et al. (2017). However, due to the use of the de-sparsified estimator in $C_{n}^{\beta}$ the set $C_{n}$ does not pick up the sparsity of $\beta^{0}$ and can easily be seen to have width of order at least $\sqrt{p \log (p) / n}$ with probability as close to one as desired. This is sub-optimal and diverges to $\infty$ in the high-dimensional setting $p \gg n$, rendering $C_{n}$ practically useless compared to the minimax rate of estimation of $X_{n+1}^{T} \beta^{0}$, which is $\sqrt{s_{0} \log (p) / n}$.

We conjecture here that it is impossible to have inference procedures that both have good $\ell_{\infty}$ and $\ell_{2}$ behaviour simultaneously due to the need for de-sparsification in the $\ell_{\infty}$-case and the need for sparse estimators that adapt to $s_{0}$ in the $\ell_{2}$-case.

Moreover, even when one uses a sparse estimator picking up the optimal rate $\sqrt{s_{0} \log (p) / n}$ is in general not achievable by confidence intervals for the prediction problem.

Using decision theoretic principles laid out in chapter 8.3 in Giné and Nickl (2016) and carefully investigating the proofs in Nickl and Geer (2013) one can prove the following result: we define the space of $p^{1-\gamma}$-sparse vectors as

$$
\Theta_{\gamma}:=\left\{\beta: \beta \in \mathbb{R}^{p}, \sum_{i: \beta_{i} \neq 0} 1 \leq p^{1-\gamma}\right\}, \quad 0<\gamma<1,
$$

and have:

Theorem 1 Suppose that $p \geq n$ and that $p^{1-\gamma}=o(n / \log (p))$ for some $0<\gamma<1$. Furthermore, assume that the $X_{i}$ are i.i.d. $\mathcal{N}(0, I)$ distributed, $i=1, \cdots, n+1$. Finally, assume that for some $0<\alpha<1 / 3$, the confidence set $C_{n}$ is honest for $X_{n+1}^{T} \beta$ over $\Theta_{\gamma}$, satisfying

$$
\sup _{\beta \in \Theta_{\gamma}} P_{\beta}\left(X_{n+1}^{T} \beta \notin C_{n}\right) \leq \alpha .
$$

Then, necessarily for any $1>\gamma_{1}>\gamma$,

$$
\sup _{\beta \in \Theta_{\gamma_{1}}} E_{\beta}\left|C_{n}\right| \geq C \min \left(n^{-1 / 4}, \sqrt{p^{1-\gamma} \log (p) / n}\right) .
$$

Specifically this implies that it is impossible to achieve adaptation, i.e. obtaining the optimal rate $\sqrt{s_{0} \log (p) / n}$ for various values of $\beta^{0}$ and $s_{0}$, in the highly sparse region with $s_{0}=o(\sqrt{n} / \log (p))$ assumed by Dezeure et al. (2017). Of course, one might say that a diameter of $n^{-1 / 4} \vee \sqrt{s_{0} \log (p) / n}$ is acceptable and is much better than the one of (1) as it shrinks to 0 . However, extending the proof of Theorem 1 using the results from Ingster et al. (2010) one sees that even this is only attainable if one assumes that the variance of the errors $\varepsilon_{i}$ is known and otherwise the rate $\sqrt{p^{1-\gamma} \log (p) / n} \wedge 1$ for an arbitrary $\gamma>1 / 2$ is the best achievable rate for the prediction problem, even if the true sparsity is much smaller. 
Other examples of functionals for which the above remarks apply include 'dense' functionals such as $\sum_{j=1}^{p} \beta_{j}^{0}$ (Cai and Guo 2017) or the $\ell_{2}$-loss $\left\|\hat{\beta}-\beta^{0}\right\|_{2}^{2}$ (Cai and Guo 2016).

We want to point out here that for matrix inference problems with rank as unknown parameter the situation is more favourable. For example, in (Carpentier et al. 2017) we consider the matrix completion problem and investigate the existence of honest and adaptive Frobenius confidence sets for the whole matrix. If 'repeated sampling' is possible we give an explicit and computable construction of such a set, even when the error variance is unknown, and thus show that in this model inference for functionals related to the Frobenius distance is possible.

To conclude, the existence of meaningful confidence statements in high-dimensional models depends highly on the statistical model, geometry of the underlying parameter space and particular aspect under consideration and requires careful case by case consideration. For practitioners the message is that extreme care has to be exercised when constructing confidence sets. Dezeure et al. should be congratulated for singling out a set of well-posed high-dimensional inference problems.

\section{References}

Carpentier A, Klopp O, Löffler M, Nickl R (2017) Adaptive confidence sets for matrix completion. Bernoulli, to appear

Cai TT, Guo Z (2017) Confidence intervals for high-dimensional linear regression: minimax rates and adaptivity. Ann Statist 45(2):615-646

Cai TT, Guo Z (2016) Accuracy assessment for high-dimensional linear regression. Preprint at https://arxiv. org/abs/1603.03474

Chernozhukov V, Chetverikov D, Kato K (2013) Gaussian approximations and multiplier bootstrap for maxima of sums of high-dimensional random vectors. Ann Statist 41(6):2786-2819

Dezeure R, Bühlmann P, Zhang CH (2017) High-dimensional simultaneous inference with the bootstrap. TEST. doi:10.1007/s11749-017-0554-2

Giné E, Nickl R (2016) Mathematical foundations of infinite-dimensional statistical methods. Cambridge University Press, Cambridge

Ingster YI, Tsybakov AB, Verzelen N (2010) Detection boundary in sparse regression. Electron J Statist 4:1476-1526

Nickl R, van de Geer S (2013) Confidence sets in sparse regression. Ann Statist 41(6):2852-2876 